\title{
Resolução de conflitos em serviços de saúde e práticas restaurativas: 0 desafio da gestão
}

\author{
Conflict resolution in health services and restaurant practices: the management challenge \\ Resolución de conflictos en los servicios de salud y las prácticas restaurativas: el desafío \\ de la gestión
}

Raquel Silva Pereira ${ }^{1 *}$, Krisla Nathane Soares dos Santos Pereira ${ }^{1}$, Gabriel Lucas Pereira Guimarães ${ }^{1}$, Emilly Jhully Correia de Paula ${ }^{1}$, Leonardo Santos Silva ${ }^{1}$, Paula Paulina Costa Tavares ${ }^{1}$.

\section{RESUMO}

Objetivo: Identificar as principais práticas do gestor na resolução de conflitos em serviço de saúde, bem como as principais dificuldades nesse processo. Métodos: Trata se de uma revisão de literatura de delineamento descritivo e abordagem qualitativa por meio de dados secundários por levantamento bibliográfico de publicações científicas entre 2010 e 2020 no Banco de dados BVS e SciELO. Resultados: A coparticipação da equipe e a capacidade de dialogar são ferramentas capazes de facilitar a resolução dos conflitos. A ausência de especialização e experiência tendem a diminuir a qualidade da gestão. É notório a existência de uma carência quanto a publicações que abordem a temática das práticas restaurativas. Considerações finais: Uma gestão eficiente possibilita que a rede de atenção básica se estruture e opere de forma resolutiva, elevando a qualidade do serviço e aliviando as demandas para os serviços especializados, diminuindo o congestionamento do sistema. Há uma defasagem nas instituições de graduação quanto ao preparo de seus discentes para atuarem como gestores.

Palavras-chave: Negociação, Enfermagem, Gestão em saúde.

\begin{abstract}
Objective: Identify the main practices of the manager in the resolution of conflicts in health services, as well as the main difficulties in this process. Methods: This is a literature review of a descriptive design and qualitative approach using secondary data through a bibliographic survey of scientific publications between 2010 and 2020 in the VHL and SciELO databases. Results: Team co-participation and the ability to dialogue are tools capable of facilitating conflict resolution. The lack of specialization and experience tends to decrease the quality of management. It is clear that there is a lack of publications on the theme of restorative practices. Final considerations: Efficient management allows the primary care network to be structured and operated in a resolutive manner, raising the quality of the service and alleviating the demands for specialized services, reducing congestion in the system. There is a gap in undergraduate institutions regarding the preparation of their students to act as managers.
\end{abstract}

Keywords: Negotiation, Nursing, Health management.

\section{RESUMEN}

Objetivo: Identificar las principales prácticas del directivo en la resolución de conflictos en los servicios de salud, así como las principales dificultades en este proceso. Métodos: Se trata de una revisión bibliográfica de diseño descriptivo y abordaje cualitativo utilizando datos secundarios a través de un relevamiento bibliográfico de publicaciones científicas entre 2010 y 2020 en las bases de datos BVS y SciELO. Resultados: La coparticipación en equipo y la capacidad de diálogo son herramientas capaces de facilitar la resolución de

${ }^{1}$ Faculdade Adventista da Bahia, Cachoeira - BA. *E-mail: raquelsil73@outlook.com 
conflictos. La falta de especialización y experiencia tiende a disminuir la calidad de la gestión. Es evidente que faltan publicaciones sobre el tema de las prácticas restaurativas. Consideraciones finales: Una gestión eficiente permite estructurar y operar la red de atención primaria de manera resolutiva, aumentando la calidad del servicio y aliviando las demandas de servicios especializados, reduciendo la congestión del sistema. Existe un vacío en las instituciones de pregrado en cuanto a la preparación de sus estudiantes para actuar como gerentes.

Palabras clave: Negociación, Enfermería, Gestión de la salud.

\section{INTRODUÇÃO}

O ambiente de trabalho no âmbito geral está sempre em constante mudança. A cada dia que se passa as exigências se tornam maiores e mais específicas, as entrevistas de emprego mais rigorosas, tudo isso com o propósito de obter uma equipe com boa funcionalidade e com um número reduzido de conflitos. Em uma frequência maior ou menor, todo ambiente de trabalho está propenso ao desenvolvimento de conflitos, e essa frequência possui uma relação direta com alguns fatores como quantidade de pessoas que compõem determinada equipe, área de atuação e/ou nível de estresse a que esses indivíduos são submetidos rotineiramente. Outro ponto que corrobora com geração de conflitos são as mudanças constantes no ambiente laboral no que se refere aos avanços tecnológicos, submetendo esses profissionais a constante tensão diante de suas responsabilidades (TORRES SC e CUNHA P, 2014).

O conflito é definido como um processo de luta, enfrentamento ou oposição entre duas ou mais partes que possuem divergência de ideias e de opiniões (FERREIRA ABH, 2010). Os trabalhadores da área da saúde são apontados por inúmeros autores como um grupo de maior propensão a desenvolver grandes conflitos, a saber pelo grau de complexidade a que estão expostos diariamente, os desafios relacionados a mudanças constantes quanto a doenças, conhecimentos inovadores em contrapartida de outros que entram em obsolescência, imprevisibilidade das ocorrências, estrutura organizacional e hierarquia de comando e subordinação a ela associada, dentre outros fatores.

A gravidade particular dos conflitos na área da saúde dificulta a resolutividade de tais conflitos, implicando diretamente na qualidade da prestação do serviço multidisciplinar oferecido nos serviços de saúde. Lidar com tais questões é uma atividade inerente ao gestor em saúde (SODER R, et al., 2018; RIBEIRO WC, 2018). É necessário que haja uma visualização frente ao que difere a liderança do gerenciamento, afinal, em tempos passados, os líderes eram agentes responsáveis tanto pela difusão das práticas de gestão quanto pelo gerenciamento da força de trabalho, estando intimamente ligado a uma posição junto ao topo da hierarquia de comando organizacional.

Entretanto, com a evolução dos conceitos é substancialmente necessário distinguir as definições, afinal, nos dias de hoje um líder pode por exemplo, ocupar um cargo significativamente inferior na hierarquia organizacional. O gerenciamento deve ser capaz de produzir ordem e consistência através do desenvolvimento do planejamento em curto prazo, organização e controle resolutivo de problemas; já a liderança está voltada a prática produtiva de mudanças e movimento nas organizações através da criação de uma visão, desenvolvendo estratégias, alinhando pessoas, e se responsabilizando por inspirar e motivar o grupo (FERREIRA MF e SILVA CFR, 2012).

A problemática das estratégias de gestão de conflito bem como as variáveis que influenciam no surgimento deles tem sido objeto de inúmeras análises no decorrer dos anos, devido a importância que essa temática exerce sobre todos os processos de trabalho, inclusive os de saúde (TORRES SC, 2014; CORRADI EZ, et al., 2008).

Criar um ambiente de trabalho prazeroso onde o relacionamento interpessoal, juntamente com a ótima assistência e o ânimo dos profissionais não sejam afetados de forma negativa, é de total responsabilidade do administrador, para que assim possa ser prestado um serviço qualificado ligado há um local favorável de trabalho para os profissionais (MARQUIS BL e HUSTON CJ. 2010). 
A literatura científica aborda inúmeras relações existentes entre as diferenças individuais dos indivíduos prestadores de serviço em setores de saúde, estilos de gestão e sua forma particular de resolver conflitos, bem como suas principais abordagens de negociação e as implicações relacionadas a essa temática (TORRES SC, 2014; SPAGNOL CA, 2011).

O presente estudo se faz substancialmente necessário baseado na necessidade existente de se conhecer os principais tipos de conflitos existentes bem como os principais fatores e fatos desencadeantes dos mesmos, para então tornar possível aos gestores desenvolverem, além de práticas restaurativas, a capacidade de se anteciparem aos conflitos mais comuns e trabalharem no desenvolvimento e/ou aprimoramento das habilidades necessárias para sua resolução. A partir do supracitado, o presente estudo possui como objetivo geral identificar as principais práticas do gestor na resolução de conflitos em serviço de saúde; e como objetivos específicos conceituar os principais tipos de conflitos, descrever as formas de gerenciamento mais utilizados, bem como os principais fatores associados ao surgimento de tais conflitos, e por fim, citar as principais dificuldades para o bom gerenciamento apontados pela literatura.

\section{MÉTODOS}

Trata se de uma revisão de literatura de delineamento descritivo e abordagem qualitativa por meio de dados secundários. Foi realizado um levantamento bibliográfico de publicações científicas no Banco de dados Biblioteca Virtual de Saúde (BVS) e também na base de dados Scientific Electronic Library Online (SciELO). Após as buscas foram encontrados por meio da leitura do título um total de 63 artigos. Para a seleção dos artigos foram adotados como critério de inclusão: artigos completos em português, no período de 2010 a 2020, que tratem das temáticas: negociação, Enfermagem e Gestão em saúde. Em seguida foram adotados como critérios de exclusão: artigos duplicadas, teses, monografias e resumos de anais. Ao final, foram selecionados 9 artigos que compõem o corpo deste estudo. O percurso de inclusões e exclusões desta revisão está disposto na Figura 1.

Figura 1 - Fluxograma das informações da pesquisa nas bases BVS e SciELO mapeando o número de registros identificados, incluídos e excluídos, e os motivos para exclusões, de acordo com o PRISMA Flow Diagram.
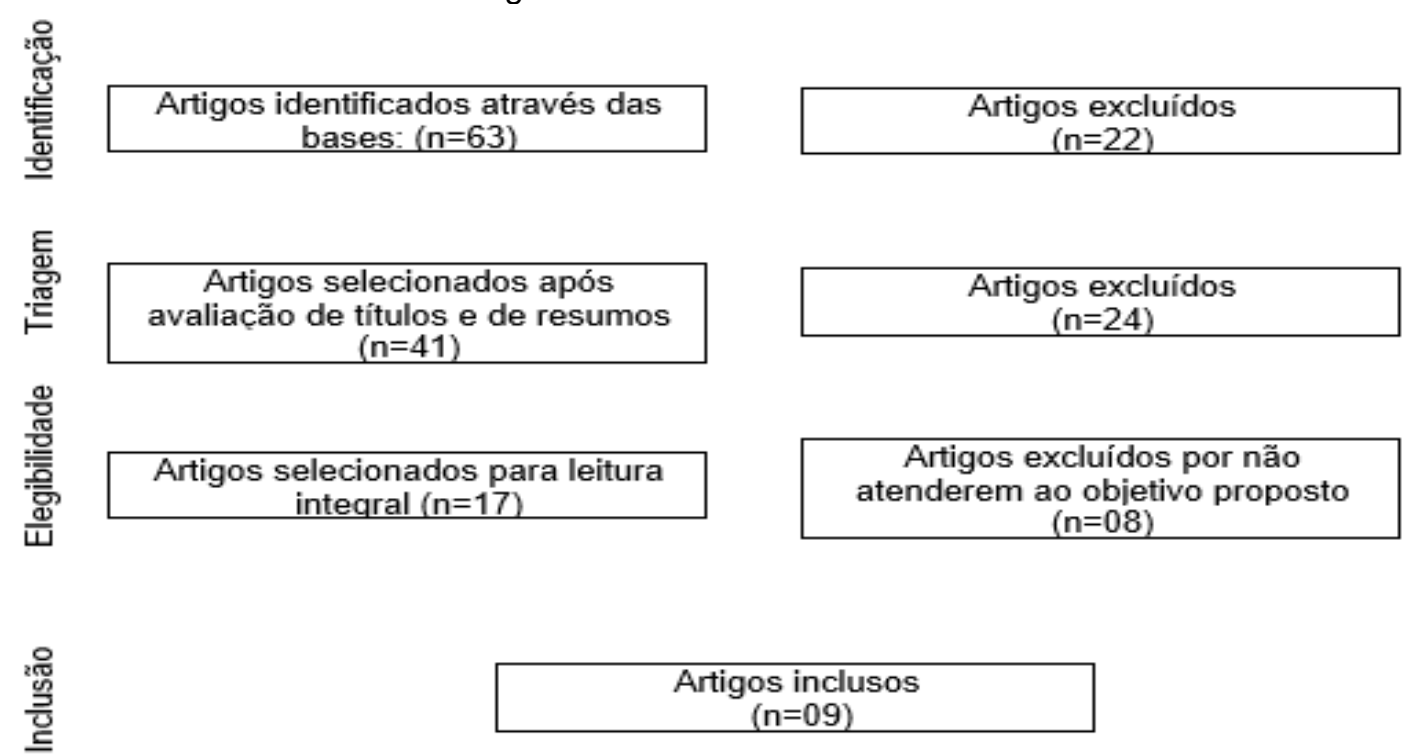

Fonte: Pereira RS, et al., 2020.

\section{RESULTADOS}

Nesta revisão foram utilizados 9 artigos, cujos temas estão dispostos no Quadro 1, sendo este referente aos autores, título dos artigos, objetivo, revista em que o mesmo foi publicado e base de dados correspondente. Dos 9 estudos selecionados, três foram encontrados na base de dados BVS, e seis dos artigos na SCIELO. Os dados das bases foram processados e organizados no Quadro 1. 
Quadro 1 - Delineamento dos artigos utilizados na revisão.

\begin{tabular}{|c|c|c|c|c|}
\hline Autores & Título & Objetivos & Revista & $\begin{array}{l}\text { Base de } \\
\text { dados }\end{array}$ \\
\hline $\begin{array}{l}\text { Silva GS, } \\
\quad \text { et al. }\end{array}$ & $\begin{array}{l}\text { Administração de } \\
\text { conflitos: análise de } \\
\text { percepções de } \\
\text { enfermeiros gerentes }\end{array}$ & $\begin{array}{l}\text { O objetivo deste estudo foi analisar as } \\
\text { percepções de enfermeiros em cargos } \\
\text { de gerência de Unidades Básicas de } \\
\text { Saúde (UBS) sobre a administração de } \\
\text { conflitos. Consta de um estudo de } \\
\text { caso qualitativo. }\end{array}$ & $\begin{array}{l}\text { R. Enferm. } \\
\text { Cent. O. } \\
\text { Min. }\end{array}$ & BVS \\
\hline $\begin{array}{l}\text { Guerra } \\
\text { ST, et al. }\end{array}$ & $\begin{array}{l}\text { Conflito na gestão de } \\
\text { enfermagem no contexto } \\
\text { hospitalar }\end{array}$ & $\begin{array}{c}\text { Este estudo objetivou analisar como os } \\
\text { conflitos se manifestam nas relações } \\
\text { interpessoais e as dimensões que } \\
\text { assumem na gestão de enfermagem } \\
\text { em hospitais. }\end{array}$ & $\begin{array}{l}\text { Rev. } \\
\text { Latino-Am. } \\
\text { Enfermage } \\
\quad \mathrm{m}\end{array}$ & BVS \\
\hline $\begin{array}{l}\text { Spagnol } \\
\mathrm{CA} \text {, et al. }\end{array}$ & $\begin{array}{l}\text { Situações de conflito } \\
\text { vivenciadas no contexto } \\
\text { hospitalar: a visão dos } \\
\text { técnicos e auxiliares de } \\
\text { enfermagem }\end{array}$ & $\begin{array}{c}\text { Objetiva analisar como o enfermeiro } \\
\text { lida com os conflitos no ambiente } \\
\text { organizacional. }\end{array}$ & $\begin{array}{l}\text { Revista da } \\
\text { escola de } \\
\text { Enfermage } \\
\text { m da USP }\end{array}$ & BVS \\
\hline $\begin{array}{l}\text { Vendemia } \\
\text { tti M, et } \\
\text { al. }\end{array}$ & $\begin{array}{l}\text { Conflito na gestão } \\
\text { hospitalar: o papel da } \\
\text { liderança }\end{array}$ & $\begin{array}{c}\text { Contextualizar o processo de } \\
\text { profissionalização da gestão hospitalar } \\
\text { e destaca os desafios para a atuação } \\
\text { das lideranças para lidar com a } \\
\text { dimensão do conflito existente entre as } \\
\text { subculturas médica, de enfermagem e } \\
\text { administrativa. }\end{array}$ & $\begin{array}{l}\text { Ciênc. } \\
\text { saúde } \\
\text { coletiva }\end{array}$ & SCIELO \\
\hline $\begin{array}{l}\text { Clarol } \\
\text { RFS, } \\
\text { Cunha } \\
\text { PFSS. }\end{array}$ & $\begin{array}{l}\text { Estratégias de gestão } \\
\text { construtiva de conflitos: } \\
\text { uma perspectiva dos } \\
\text { profissionais de saúde }\end{array}$ & $\begin{array}{c}\text { O objetivo geral do presente artigo } \\
\text { prende-se com o estudo dos conflitos } \\
\text { que surgem em meio hospitalar, bem } \\
\text { como das estratégias de resolução de } \\
\text { conflitos utilizadas pelos profissionais } \\
\text { de saúde para a gestão dos mesmos e } \\
\text { a aplicação da Mediação de conflitos } \\
\text { na área da saúde. } \\
\end{array}$ & $\begin{array}{l}\text { Psic., } \\
\text { Saúde \& } \\
\text { Doenças }\end{array}$ & SciELO \\
\hline $\begin{array}{l}\text { Santos } \\
\text { MC, } \\
\text { Bernarde } \\
\text { S A. }\end{array}$ & $\begin{array}{l}\text { Comunicação da equipe } \\
\text { de enfermagem e a } \\
\text { relação com a gerência } \\
\text { nas instituições de saúde }\end{array}$ & $\begin{array}{l}\text { Tal estudo objetiva analisar as } \\
\text { contribuições das pesquisas } \\
\text { produzidas acerca da comunicação na } \\
\text { gerência de enfermagem, tomando por } \\
\text { base as publicações em periódicos } \\
\text { nacionais. }\end{array}$ & $\begin{array}{l}\text { Rev. } \\
\text { Gaúcha } \\
\text { Enferm. }\end{array}$ & SciELO \\
\hline $\begin{array}{l}\text { Frederico- } \\
\text { Ferreira } \\
\text { M, Silva } \\
\text { CFR. }\end{array}$ & $\begin{array}{l}\text { Reformas da gestão na } \\
\text { saúde: desafios que se } \\
\text { colocam aos enfermeiros }\end{array}$ & $\begin{array}{l}\text { Pretendeu-se conhecer quais os } \\
\text { desafios, considerados pelos } \\
\text { enfermeiros, que se colocam à } \\
\text { profissão de Enfermagem perante } \\
\text { estas mudanças. }\end{array}$ & $\begin{array}{l}\text { Rev. Enf. } \\
\text { Ref. }\end{array}$ & SciELO \\
\hline $\begin{array}{l}\text { Peduzzi } \\
\text { BGCM, } \\
\text { Ayres } \\
\text { JRCM. }\end{array}$ & $\begin{array}{l}\text { Concepções e tipologia } \\
\text { de conflitos entre } \\
\text { trabalhadores e gerentes } \\
\text { no contexto da atenção } \\
\text { básica no Sistema Único } \\
\text { de Saúde. } \\
\end{array}$ & $\begin{array}{c}\text { Este estudo objetivou analisar } \\
\text { percepções de conflito entre } \\
\text { trabalhadores e gerentes de unidade } \\
\text { básica de saúde e apresentar tipologia } \\
\text { de conflitos vivenciados no trabalho. }\end{array}$ & $\begin{array}{l}\text { Cad. } \\
\text { Saúde } \\
\text { Pública }\end{array}$ & SciELO \\
\hline $\begin{array}{l}\text { Claro } \\
\text { RFS, } \\
\text { Cunha } \\
\text { PFSS. }\end{array}$ & $\begin{array}{l}\text { Gestão de conflito na } \\
\text { área da saúde: uma } \\
\text { proposta de reflexão. }\end{array}$ & $\begin{array}{l}\text { Refletir sobre a gestão de conflito, na } \\
\text { área da saúde. }\end{array}$ & Arq Med & SciELO \\
\hline
\end{tabular}

Fonte: Pereira RS, et al., 2020. 


\section{DISCUSSÃO}

Foi apontado com o estudo em questão algumas das principais estratégias gerenciais utilizadas na resolução dos conflitos, dentre elas podemos ressair como preponderantes: dialogar com a equipe; mostrar resolutividade no enfrentamento dos conflitos; coparticipação da equipe na tomada de decisão; ser imparcial e priorizar a qualidade do serviço; manter postura profissional e manejar as situações individualmente (LIMA SB, et al., 2014; SANTOS MC e BERNARDES A, 2010). A coparticipação da equipe junto a capacidade de dialogar é uma ferramenta capaz de implementar inúmeras mudanças, dividir ideias e informações se desenvolver habilidades e maturidade dos liderados (SANTOS MC e BERNARDES A, 2010).

Além dessas estratégias, algumas ações como promoção de espaços em que os profissionais possam expressar seus sentimentos e expor os conflitos que estão acontecendo dentro da equipe é imprescindível para a resolutividade, afinal, essas situações nem sempre são explícitas e na maioria das vezes, fica restrito à alterações no tom de voz, indiferença e agressividade entre os profissionais, tornando o ambiente de trabalho tenso e gerando um alto nível de estresse, dificultando ainda mais as relações interpessoais e interferindo significativamente na realização do trabalho e levando a um clima hostil em que as pessoas deixam de lado a sinceridade e começam ofender o outro (LIMA SB, et al., 2014; SANTOS MC e BERNARDES A, 2010; FERREIRA MF e SILVA CFR, 2012).

No que se refere à Atenção Básica, os desafios da gestão do cuidado a partir da perspectiva de equipes de enfermagem são normalmente apresentados em três categorias: morosidade da gestão pública; sobrecarga de trabalho; e fragmentação e descontinuidade da assistência. A morosidade da gestão pública resulta na má utilização de recursos físicos e humanos que interfere diretamente nas ações do sistema de saúde, promovem atraso nas licitações e consequentemente em toda a assistência. Quando não há uma boa distribuição de trabalho da parte do gestor, o profissional acaba por se sobrecarregar de maneira excessiva, não sendo possível prestar o cuidado e a escuta que seriam possíveis em uma situação onde houvesse uma divisão correta das atividades entre os profissionais. Para além disso, a ausência de cobertura 100\% pelas ESF e a falta de referência e contra referência principalmente na alta hospitalar, acabam por gerar fragmentação e descontinuidade nos serviços (SODER R, et al., 2018).

A ausência de conhecimento do gestor referente a gama de pessoas que estão em constante interação e sobre o nível de estabilidade emocional que cada um possui, deixando de lado o reconhecimento de que a pessoa vem antes do profissional, é caracterizado como um dos grandes empecilhos quanto a escolha da melhor estratégia para resolução de conflitos quando estes surgem (GUERRA ST, et al., 2011).

Entre as habilidades necessárias à um gestor, encontra-se a capacidade de conversar com os membros da equipe sobre os conflitos como mediador imparcial, compreensivo e capaz de priorizar a qualidade do serviço e da assistência prestada, tratando assim, todos os funcionários de forma igualitária, sem privilégios, embora em algumas situações isso pode gerar descontentamento por uma parte, por não sentir que suas necessidades foram colocadas em primeiro plano, gerando a ideia de injustiça, e de falta de habilidade e capacidade do enfermeiro gestor para a resolução daquele conflito (LIMA SB, et al., 2014; SPAGNOL CA, et al., 2010; CUNHA P, et al. 2013; VENDEMIATTI M, et al., 2010).

Apesar dos conflitos serem vistos em sua maioria como situações corriqueiras no ambiente de trabalho, não são considerados uma tarefa simples, por sempre envolverem indivíduos "tensos" e "interesses distintos" (CLARO RFS e CUNHA PFSS, 2017; CARVALHO BG, et al., 2014; SILVA GS, et al., 2012). O atraso nos processos de trabalho foi apontado por vários autores dos artigos encontrados, como o principal agente para a identificação da falta de estrutura, atualização e capacitação profissional.

Podemos evidenciar essa afirmação pelo fato de que várias Unidades de Saúde obtém equipamentos e materiais de alta tecnologia disponíveis para os seus usuários, mas em contrapartida, não dispõem de profissionais que sejam devidamente capacitados para manusear e manter da maneira correta 0 funcionamento destes aparelhos/equipamentos. No entanto por outro lado, ainda existem bastante processos que não são tão úteis quanto deveriam ser, por estarem ultrapassados para o contexto daquela unidade. 0 despreparo das unidades no que diz respeito a falta de material e escassez de equipamentos, associadas à grande demanda apresentada pelos usuários, também acabam por gerar situações estressantes e 
conflituosas, devido à disputa para utilização dos materiais necessários para cumprimento de tarefas (LIMA SB, et al., 2014; SANTOS MC e BERNARDES A, 2010).

Percebe-se que os conflitos tendem a surgir principalmente durante a interação interpessoal cotidiana e seu desencadeamento está relacionado à forma como cada um conduz e responde às interações do dia a dia. Outros fatores relacionados ao desencadeamento dos conflitos mantém estreita relação com a influência do ambiente organizacional, à falta de empatia de alguns colaboradores, divergências de opiniões, diferenças culturais e religiosas, dificuldade de se relacionar, problemas particulares, conduta profissional de desvalorização e descompromisso com o trabalho, julgamentos relacionados a índole, falta de responsabilidade e cooperação com outros membros da equipe e fofoca (SANTOS MC e BERNARDES A, 2010; VENDEMIATTI M, et al., 2010; CLARO RFS e CUNHA PFSS, 2017; CARVALHO BG, et al., 2014; SILVA GS, et al., 2012).

Dentre os fatores que dificultam o manejo dos conflitos prevalentes identificados estão: sensação de impunidade frente a algumas situações, conflitos passados que não se resolveram de forma satisfatória e tendem a agravar a situação posterior, sobrecarga de responsabilidade em cima do enfermeiro gestor, debilidades da estrutura e capacitação profissional e conflito de interesses individuais e do serviço (SODER R, et al., 2018; FERREIRA MF e SILVA CFR, 2012; CLARO RFS e CUNHA PFSS, 2017; ANTONIASSI CP, et al., 2019; JUNQUEIRA LA, 1990).

A implementação de práticas restaurativas é apontada como um gerador de resultados positivos, quando colocado em prática dentro das instituições, visa o resgate de relações interpessoais que foram perdidas por conta de conflitos passados, além de possibilitar ao gestor ficar em posição semelhante aos demais durante o desenvolvimento dessas práticas, criando uma abertura para o bom relacionamento entre gestor e colaborador, o que possibilita ainda mais que as opiniões sejam expressadas sem imposições ou receios. A ausência de uma divulgação ampla do organograma institucional dificulta que os indivíduos envolvidos nos processos conflituosos saibam a quem se reportar e informar sobre o conflito logo que este surge, dificultando ainda mais a sua resolução na medida em que este se prolonga (VENDEMIATTI M, et al., 2010).

A ausência de especialização para capacitação em gerência é comumente apontada como uma questão reconhecida como necessária, porém, a ausência de remuneração justa frente ao processo faz com que os profissionais não invistam nesse tipo de aperfeiçoamento (FERREIRA MF e SILVA CFR, 2012). Questões relacionadas ao trabalho, formação e preparo são abordadas como forma de avaliar e compreender as condições em que cada profissional se apresenta e como isso interfere diretamente na sua tomada de decisão.

A falta de preparo do enfermeiro para lidar com os conflitos organizacionais, é associado à diversos fatores como: pouca ou nenhuma experiência profissional, falta de interação entre gestor e equipe, ausência de diálogo, estratégias comunicativas defasadas, falta de autoridade, de bom senso e de sabedoria, fazem com que o enfermeiro se sinta impotente diante da situação, preferindo então a fuga do conflito ao invés de sua resolução. Portanto, a conduta a ser adotada pelo enfermeiro diante de uma situação conflituosa, será influenciada por sua postura enquanto gerente e as habilidades pessoais que o mesmo possui no processo de negociação e utilização de estratégias (SODER R, et al., 2018; SANTOS MC e BERNARDES A, 2010).

Embora existam alternativas mais usadas e indicadas para resolução dos conflitos, o estilo de conduzir a situação será determinado pela personalidade de cada gerente, os personagens envolvidos e o tipo de situação (SPAGNOL CA, et al., 2010).

Dentre todas as variáveis administrativas, gerenciar pessoas é apontado como a mais difícil e importante para o êxito da organização, com destaque para o manejo e resolução de problemas (GUERRA ST, et al., 2011).

A administração de conflitos mediada pelos enfermeiros mostra que a formação na graduação é insuficiente para sua atuação no sentido de lidar com a complexidade das demandas inerentes ao tema. No entanto, apesar de ser apontada como uma prática comum a diversas instituições, a inserção precoce de enfermeiros em cargos de gerência sem que estes possuam experiência e também desprovidos de conhecimentos teóricos considerados suficientes, em muitas situações influencia negativamente na 
capacidade de resolver conflitos, prejudicando ainda mais o processo de resolução do conflito. Para além disso, a incapacidade de se administrar a equipe também diz respeito à falta de qualificação complementar para desempenhar o trabalho gerencial, desencadeando outros problemas, como a falta de confiança da equipe de trabalho (SILVA GS, et al., 2012).

Os estudos evidenciaram que a grande maioria dos enfermeiros se tornaram gerentes por nomeação contrária à sua vontade e sem a menor noção de fatores e competências imprescindíveis para desenvolvimento desta função. A falta de preparação é apontada como um problema decorrente de uma falha na oferta de conhecimentos e práticas gerenciais pelas instituições de graduação, particularmente quanto à capacidade de dialogar, desenvolvimento de estabilidade emocional, capacidade de igualar diferenças entre os profissionais, seja na própria equipe ou no grupo de outros trabalhadores da organização e também de lidar com as expectativas do grupo, que pode estar relacionada à escala de trabalho, preferências por especialidades ou questões particulares que causam desajustes nas obrigações familiares e atividades profissionais (GUERRA ST, et al., 2011).

Grande parte dos conflitos fogem da governabilidade dos gestores, por estar relacionada a questões políticas, financeiras, ideológicas e individuais, provocado nos gestores a sensação de que sua atuação na gestão é irrelevante e substituível (SILVA GS, et al., 2012).

Gerentes que apresentam dificuldades de liderar a equipe, acabam tendo repercussões na sua capacidade de negociação, fazendo com que necessite recorrer à observação da hierarquia para a resolução da situação, não se considerando a vontade dos outros envolvidos. A recorrência às relações de poder para a resolução de conflitos é na maioria das vezes decorrente de uma falha no processo de negociação e resolução de um impasse (CARVALHO BG, et al., 2014; SILVA GS, et al., 2012).

Situações de conflito que envolvem os usuários normalmente estão associados a limitação de oferta de serviços e são permeados por muita falta de compreensão, o que acaba por dificultar a resolução. Esse tipo de conflito tem o seu sucesso de negociação normalmente determinado pela existência ou não do vínculo de confiança criado pelo gerente (CLARO RFS e CUNHA PFSS, 2017; SILVA GS, et al., 2012).

Os que conflitos que envolvem médicos acabam por limitar a autonomia do enfermeiro gerente, pela forma como os mesmos são considerados pelos demais profissionais de saúde e por possuírem uma idéia de superioridade frente ao enfermeiro gestor, cuja autonomia se mostra limitada, nessa situação (VENDEMIATTI M, et al., 2010; SILVA GS, et al., 2012).

As estratégias buscam esclarecer os fatos que ocorreram, resolução dos problemas, harmonia e colaboração entre os membros da equipe de enfermagem, são descritas na literatura como meios para conduzir os conflitos no ambiente de trabalho, e podem ocorrer por meio de: Confrontação - onde os problemas são resolvidos por meio de uma abordagem direta entre as partes envolvidas, sendo este o método clássico utilizado por gestores; Abrandamento - quando os interesses comuns são enfatizados, procurando diminuir as diferenças entre os envolvidos; Negociação - ambas as partes abrem mão de algo; Evitar o conflito - evitando polêmicas e manipulando as condições organizacionais e emocionais; Retirada - quando o gerente demora para responder a questão e deixa que se resolva por si, evitando o conflito ao invés de enfrentá-lo; e Peso da autoridade - quando uma autoridade competente toma a derradeira decisão (SANTOS MC, BERNARDES A, 2010; SILVA GS, et al, 2012).

\section{CONSIDERAÇÕES FINAIS}

A introdução precoce de enfermeiros despreparados na função de gestor é considerada um dos fatores que mais dificultam os processos de negociação e resolução de conflitos. Há uma grande necessidade de se investir em especialização em gestão para que os gestores desempenhem seu papel de forma funcional, entretanto, a ausência de reconhecimento e remuneração frente a especialização acaba por diminuir a procura e interesse. A falta de abordagens teóricas e práticas em gestão é um problema existente nas instituições de graduação que possuem seu foco no ensino de práticas terapêuticas, porém uma defasagem 
no quesito gestão, mesmo esse último sendo de grande importância na atuação do enfermeiro. A incapacidade de resolver conflitos consiste em uma questão de grande impacto frente ao bom desenvolvimento de todo o sistema de prestação de serviços de saúde.

\section{REFERÊNCIAS}

1. ANTONIASSI CP, et al. Práticas restaurativas na gestão de uma equipe de Estratégia Saúde da Família: relato de experiência em Pato Branco, PR. Saúde debate, 2019; 43(6): 0103-1104

2. CARVALHO BG, et al. Concepções e tipologia de conflitos entre trabalhadores e gerentes no contexto da atenção básica no Sistema Único de Saúde (SUS). Cad. Saúde Pública, 2014; 30(7):1453- 1462.

3. CLARO RFS, CUNHA PFSS. Estratégias de gestão construtiva de conflitos: uma perspetiva dos profissionais de saúde. Psic., Saúde \& Doenças, 2017; 18(1):55-68.

4. CORRADI EZ, et al. O gerenciamento de conflitos entre a equipe de enfermagem. Rev. Cogitare Enfermagem. 2008, Curitiba 13(2) 184-193.

5. CUNHA P, et al. Gestão de conflitos na área da saúde: uma proposta de reflexão. Arq. Med. 2013; 27(3):132-134.

6. FERREIRA ABH. Mini Aurélio: o dicionário da língua portuguesa. 8a Ed. Curitiba: Positivo, 2010.

7. FERREIRA MF, SILVA CFR. Reformas da gestão na saúde: desafios que se colocam aos enfermeiros. Rev. Enf. Ref. 2012; 3:85-93.

8. GUERRA ST, et al. Conflict in nursing management in the hospital context. Rev. Latino-Am. Enfermagem, 2011; $19(2): 1-8$.

9. JUNQUEIRA LA. Gerência dos Serviços de Saúde. Cad. Saúde Pública, 1990; 6(3):247-259.

10. LIMA SBS, et al. Conflitos gerenciais e estratégias de resolução pelos enfermeiros gerentes. Rev de Enfermagem da UFSM, 2014 4(2):419-428

11. MARQUIS BL, HUSTON CJ. Administração e Liderança em Enfermagem - teoria e prática. 6 edição Porto Alegre: Artmed, 2010.

12. RIBEIRO WC. A mediação como meio de resolução de conflitos na área de saúde.R. Dir. sanit. 2018, 15(3) 62-73.

13. SANTOS MC, BERNARDES A. Comunicação da equipe de enfermagem e a relação com a gerência nas instituições de saúde. Rev. Gaúcha Enferm. 2010, 31(2):359-366.

14. SILVA GS, et al. Administração de conflitos: análise de percepções de enfermeiros gerentes. R. Enferm. Cent. O. Min. 2012, 2(3):358-368.

15. SODER R, et al. Desafios da gestão do cuidado na atenção básica: perspectiva da equipe de enfermagem. Enferm. Foco, 2018, 9(3):1-5.

16. SPAGNOL CA. Conflito organizacional: considerações teóricas para subsidiar o gerenciamento em enfermagem. Rev. Ciência, Cuidado E Saúde, 2011, 9(4), 822-827.

17. SPAGNOL CA, et al. Situações de conflito vivenciadas no contexto hospitalar: a visão dos técnicos e auxiliares de enfermagem. Rev. esc. enferm. USP, 2010, 44(3):803-811.

18. THOFEHRNL MB, et al. Teoria dos vínculos profissionais: um novo modo de gestão em enfermagem. Rev. Texto e Contexto: Enfermagem. 2006, 15(3) 1-8.

19. TORRES SC, CUNHA P. Gestão de conflitos em uma organização da área da saúde em Portugal. Ciência e cognição, 2014, 19(3):384-392.

20. VENDEMIATTI M, et al. Conflito na gestão hospitalar: o papel da liderança. Ciênc. saúde coletiva, 2010, 15(1):1-14. 